\title{
REVIEW
}

\section{Peanut allergy prevention: Current evidence and implications for clinical practice}

\author{
Laura Allen BHSc \\ Class of 2019, Faculty of Medicine, Dalhousie University, Halifax, NS, Canada
}

\begin{abstract}
In the last decade there has been a major shift in clinical practice guidelines from the avoidance of allergenic foods for the purpose of preventing allergy development toward their early introduction before six months of age. The purpose of this article is to review current evidence for this change in recommendation and to evaluate its implications for clinical practice. A literature review and analysis were conducted focusing on key clinical studies that influenced this change in practice over the past decade. The main findings supported the early introduction of peanut to the diet and highlighted two areas of uncertainty in the literature that have implications for adopting the new guidelines in clinical practice. First, current evidence lacks a clear definition of "high risk" for determining allergic predisposition in infants; and second, defining the ideal timing, amount, and frequency with which peanut and peanut containing foods are introduced into a child's diet is not yet established in the literature. The principal conclusion underscored the importance of early and continual exposure to peanut regardless of risk of atopy.
\end{abstract}

TE-mediated food allergies in childhood are common

Iin North America and peanut allergy alone affects $1-2 \%$ of children in Canada. ${ }^{1}$ The gastrointestinal tract is exposed daily to numerous dietary proteins. The vast majority are tolerated through suppression of cellular or humoral responses: a process known as oral tolerance. Food hypersensitivity results when tolerance to a given dietary antigen is not established, or breaks down. Suppression involves signaling by an array of antigen presenting cells, dendritic cells, and regulatory $\mathrm{T}$ cells, as well as lymphocyte deletion. Factors including antigen properties, route of exposure, genetics, and age of the host all contribute to the development of oral tolerance. Although the standard of care for patients with food allergies was largely based on avoidance of the trigger, increased understanding of the mechanisms involved in tolerance now support its induction. ${ }^{18}$

Current evidence on the primary prevention of peanut allergy is limited and changing. There has been a major shift in clinical guidelines over the last decade from the avoidance of peanut containing foods to early dietary exposure of these foods in infants. Evidence-based clinical practice guidelines such as the Rourke Baby Record (RBR) aim to reflect the changing literature. The most recent 2014 RBR recommends against the delayed introduction of common allergenic foods after 4-6 months of age even for infants at risk of atopy. ${ }^{2}$ For health practitioners, the clinical implications of how to best manage high risk infants and counsel parents are complex. These challenges include discrepancies in the literature on the definition of high risk and uncertainty about the optimal dose and duration of peanut exposure once introduced. ${ }^{3}$ The aim of this article is to review current evidence surrounding the new recommendations and to evaluate their implications for clinical practice.

\section{Background}

The RBR is an evidence-based health supervision guide for primary health practitioners caring for children in the first five years of life. The RBR is endorsed by the Canadian Pediatric Society (CPS), the College of Family Physicians of Canada (CFPC), and Dieticians of Canada (DC). ${ }^{2}$ Recommendations made by the RBR are widely referenced by Canadian health practitioners, and therefore play an important role in implementing preventative health strategies in Canada. The most substantial revisions to the 2014 RBR are related to nutrition. In regards to the primary prevention of peanut allergies, the RBR recommends that infants be introduced to solid foods when they show signs of readiness (timing might range from a few weeks before, to just after 6 months of age), and that these foods include common allergenic foods such as peanut regardless of an infant's risk of atopy. ${ }^{2}$ This recommendation is consistent with current national guidelines found in Health Canada's Nutrition for Healthy Term Infants. ${ }^{2}$ By contrast, past position statements held by the RBR, CPS, and American Academy of Pediatrics (AAP) as recently as 2000 condoned the delayed introduction of peanut and tree nuts until 3 years of age in high risk infants. ${ }^{4}$ This position was not reversed until evidence emerged in 2008 suggesting the potential benefits of supporting early rather than delayed peanut exposure. ${ }^{4}$ At the time, no formal recommendations on the optimal time to introduce peanut were made, but delayed introduction was no longer recommended. ${ }^{4}$ The updated 2014 RBR recommendation is based on a growing body of evidence that supports early and continuous exposure to allergenic foods, including peanut, as a means of allergy prevention., ${ }^{4,5}$

The prevalence of peanut allergy is known to be 
Peanut allergy prevention

highest in some Westernized countries such as the UK, USA, Canada, and Australia (around 1-2\%), and lowest in Asian countries where peanut is not on the list of common allergens in children. ${ }^{6}$ The overall rate of atopic disease in developed and underdeveloped countries is rising, with food allergy being strongly associated with other atopic disorders. ${ }^{5-7}$ In developed countries, about $50 \%$ of children with a food allergy have atopic dermatitis, 40\% have asthma, and about 30\% have allergic rhinitis. ${ }^{5,7}$ In the context of rising rates of atopic disease and emerging evidence on allergy prevention, it is important for clinicians to understand the clinical implications of these changing guidelines.

\section{Methods}

Searches for peer-reviewed published journal articles were conducted using Google Scholar, Cochrane Library, PubMed, and Dalhousie University online databases in the areas of Health Sciences and Medicine. All searches were limited to research with humans published in English. Keywords included peanut allergy, early introduction, prevention, high risk, atopy, sibling, parent, allergenic foods, and dietary exposure. Approximately, 1,200 articles were identified by keyword search. Seventeen articles were reviewed in depth. These articles focused on the frequency of peanut allergy development in high risk and non-high risk children following early or late exposure to peanuts, and in some cases, to other allergenic foods such as grains, nuts, fish, and egg. One populationbased study looked at the impact of family history on allergy development. Selection criteria included articles that are recent, focus on peanut, and are preferably meta-analyses, randomized control trials, populationbased studies, or literature reviews. Current position statements from organizations including the Canadian Society of Allergy and Clinical Immunology, CFPC, DC, RBR, and CPS were also included. Exclusion criteria were met by studies not published in English.

\section{Results}

The reviewed articles collectively favoured the early introduction of peanut for the purpose of avoiding allergy development in infants. Overall, the evidence highlighted the importance of accepting new guideline recommendations and consistently identified two major implications for adapting these recommendations to clinical practice. First, it is important that health practitioners understand the meaning of "high risk" so that they can identify infants who may be considered high risk in order to counsel patients appropriately. ${ }^{3}$ Second, the evidence is still insufficient in terms of how to advise parents with regards to the optimal timing and frequency with which to introduce peanut to their child's diet. ${ }^{3}$

\section{The definition of "high risk"}

The definition of "high risk" is multifaceted and evolving in the literature. An infant at high risk for developing a food allergy has historically been defined as one having one first degree relative (a sibling or parent) with an allergic condition such as atopic dermatitis, a food allergy, asthma, or allergic rhinitis, with equal weighting given to any allergic disease. ${ }^{3}$ On the basis of limited evidence, parental history of atopy has long been considered a risk factor for food allergy development and has been consistently used in food allergy prevention trials since the 1980s. ${ }^{3}$ Another finding based on limited evidence is that the younger sibling of a peanut-allergic child has a slight (7\%) increased risk over baseline of developing a peanut allergy. $^{3}$

More recent studies have identified other risk factors for classifying infants at high risk for developing peanut allergy. Studies of note include the Learning Early About Peanut Allergy (LEAP) trial published in 2015 that randomized 640 high-risk 4-11 month olds in the UK to either consume peanut 3 times per week or avoid peanut entirely for the first five years of life. In the LEAP trial, high risk was defined as having either an egg allergy or severe eczema that were both strictly specified. ${ }^{10}$ In the peanut-avoidance group, $17.2 \%$ had a food-challenge proven peanut allergy by 5 years of age, compared to $3.2 \%$ in the peanut-consuming group. ${ }^{10}$ The LEAP trial did not take into account sibling or parental history of allergic disease when assessing the risk of peanut allergy development, yet results corresponded to a $14 \%$ absolute risk reduction with regular peanut exposure. ${ }^{10}$ Although no comparative studies using the same criteria for defining high risk have been performed, the LEAP trial strongly supports the validity of considering risk factors outside of family history when defining risk. ${ }^{10,11}$

HealthNuts, a large population-based study of 5,276 one-year-old infants measured the prevalence of oral food challenge-confirmed allergy. Associations between the development of peanut allergy and a family history of allergic conditions were assessed by multiple logistic regression. Results revealed, 70\% of participants had a positive family history and that having two or more allergic family members was strongly predictive of peanut allergy in the child, while having only one family member was not. ${ }^{12}$ Both the results of the HealthNuts and LEAP studies challenge the clinical relevance of defining high risk as having only one family member with an allergic condition, as has been historically accepted. 


\section{The introduction of peanut to the diet}

There is no known benefit to delaying the introduction of allergenic foods, including peanut. ${ }^{14}$ However, the optimal timing for their initial introduction to the infant diet is not well defined in the literature. ${ }^{3}$ The 2014 RBR recommendation of 4-6 months of age for the introduction of solid (including allergenic) foods is a step toward further specification. This recommendation is based on several studies primarily of level I and II evidence. ${ }^{2}$ Studies are now beginning to demonstrate the benefits of both early introduction and repeated exposure to peanut for preventing allergy development, as well as the potential harm in delaying introduction. $^{13,14}$ One observational study reported a ten-fold higher prevalence of peanut allergy among Jewish children living in the UK who scarcely were exposed to peanut, compared to Jewish children living in Israel where peanut was consumed regularly from early on in life. Similarly, a small study assessed the association between grain exposures (including wheat, barley, rye, and oats) and the development of wheat allergy. This study found that delaying the introduction of grains until after 6 months of age was not protective and may have increased the risk of developing wheat allergy.

The evolving and expanding literature raises many questions. From a clinical standpoint, knowing the amount and frequency of peanut exposure necessary to avoid allergy development is key, as is the question of whether to counsel patients differently according to atopic risk. The frequency of peanut exposure and the absolute amount necessary to prevent allergy development remain to be determined.,17 However, a recent study found that the early introduction of egg in smaller amounts in baked goods or in cooked form may be beneficial for preventing allergy. ${ }^{4}$ A similar study found an increased risk of sensitization to egg at 5 years of age following food-specific serum IgE testing if egg had been introduced after 10.5 months of age. ${ }^{4,13}$ The LEAP trial also provides evidence as to the questions of quantity and frequency. Participants in the peanut exposure group consumed a total of 6 grams of peanut protein, the equivalent of 24 grams of peanuts or 3 spoons of peanut butter per week. ${ }^{10}$ The resulting $14 \%$ absolute risk reduction suggests that their chosen amount and frequency of peanut exposure was effective in protecting against allergy development. These studies demonstrate that regular peanut exposure may be important for the development of tolerance. More importantly, the LEAP trial demonstrates that early and continuous peanut exposure can be successfully carried out in a high risk population. ${ }^{10,16}$

Current guidelines are in direct opposition to the established practice of peanut avoidance. In this context, parental attitudes toward current recommendations may have a significant role in determining a child's frequency of exposure to peanut. Parental adherence to current recommendations is an important factor in the clinical management of high risk infants. ${ }^{3}$ Parents with one or more children with a known peanut allergy may be especially resistant to introducing peanut to a younger sibling. Similarly, continued exposure may be less likely among these parents. ${ }^{3}$ While evidence surrounding the frequency of peanut exposure necessary to avoid allergy development is not well established, evidence stressing the potential harm caused by avoidance of allergenic foods is substantial and should be considered when counseling parents of high risk and non-high risk infants.

\section{Discussion}

The issue of counseling parents on the early introduction of peanut is multifaceted. While the literature is changing, gaps in knowledge exist. Parental attitudes toward peanut avoidance, may present challenges to implementing current guidelines to clinical practice. Certainly, evidence has shown that the delayed introduction of allergenic foods does not prevent allergic disease. The 2014 RBR reflects this evidence and has an influential role in allergy prevention on a population level. Canadian primary care providers can be confident in counseling against the delayed introduction of peanut. In addition, evidence suggests that the early introduction of peanut is protective against the development of an allergy. ${ }^{10,17}$ Furthermore, regular exposure to peanut seems to have a role in maintaining tolerance. ${ }^{17}$ It is on the basis of less evidence, but with growing support in the literature, that primary care providers can promote the introduction of allergenic foods between 4-6 months of age. While the LEAP study provides level I evidence in support of the introduction of peanut in high risk infants between 4-11 months old, more research is required to establish a definition of high risk and to determine the ideal age of peanut exposure. Adherence in the LEAP trial was $92 \%$, with those infants randomized to consume peanut consuming between 6.7-8.8 grams per week during the first two years of the trial. ${ }^{11}$ However, the study did not address the minimal length of treatment needed to introduce tolerance. ${ }^{11}$ Collectively, evidence compliments the findings of the LEAP trial in regards to the benefits of early and continuous peanut exposure. The current physiological understanding of sensitization and immunological tolerance in the medical community also favour the early introduction of peanut over its avoidance. ${ }^{8}$

Clearly, the recent shift in evidence has not undermined the value of clinical discretion in 
addressing allergy prevention in high risk infants. The value of case-by-case assessment is apparent for the identification of high risk infants and for assessing parental attitudes. There is significant room for clinical discretion in identifying infants who should be considered high risk, but evidence suggests that both family history and existing allergic conditions together are most indicative of peanut allergy development. It is likely that having only one immediate family member with an atopic condition is less predictive of allergic development than is having two or more. Evidence supports the clinical value of determining an infant's risk for atopy particularly in terms of having a known atopic condition. Regardless of atopic risk, current recommendations support the introduction of allergenic foods before six months of age.

Infants thought to be at a higher risk for developing peanut allergy due to a known sibling allergy may be less likely to have consistent exposure to peanut. ${ }^{3}$ Despite new evidence in support of the early introduction of peanut, past recommendations encouraging the delayed introduction of peanut have had a lasting impact. Both parental hesitancy and lack of peanut in the home may lead to reduced peanut exposure, underscoring the importance of patient education. Therefore, the role of patient-provider discussion may be more pronounced in the case of high risk infants. The role of counseling is emphasized for parents of high risk infants, as the degree of peanut exposure likely influences sensitization and tolerance. Reluctance to introduce peanut early may factor into decision-making more often for parents of children with existing atopic conditions or with a sibling who has a known peanut allergy. ${ }^{8}$ Such cases may benefit from referral to an allergist for food challenge testing. ${ }^{8}$ However, the CPS does not encourage general routine skin or specific IgE blood testing due to the high rate of false positive results. ${ }^{8}$ As recommended by the 2014 RBR, prevention through early introduction of peanut is the primary focus.

\section{Conclusion}

There has been a recent shift in evidence based practice regarding peanut allergy prevention. Congruent with the 2014 RBR, formal recommendations promote the introduction of peanut between 4-6 months regardless of risk of atopy. Delayed introduction of peanut is no longer standard practice, and may increase the risk of allergy development. A primary care provider may refer to an allergist for the diagnosis of food allergy in patients with severe atopic conditions. ${ }^{8}$ However, family history alone is likely not a strong predictor of food allergy development. The role of counseling parents on the changing evidence is particularly important for the prevention of peanut allergy in high risk infants. Regular exposure is likely to be an important factor in the maintenance of peanut tolerance and allergy prevention. Future directions should include further study to quantify the amount of exposure necessary to induce tolerance and to better understand the relevance of family history and existing atopic conditions in determining allergic predisposition.

\section{Acknowledgements: Dr. Fiona Bergin MD}

Assistant Professor, Department of Family Medicine,

Dalhousie University, Halifax, NS, Canada

\section{References}

1. Sicherer SH, Sampson HA. Food allergy: epidemiology, pathogenesis, diagnosis, and treatment. J Allergy Clin Immunol 2014;133(2):291-307.

2. Riverin B, Li P, Rourke, L, Leduc D, Rourke J. Rourke Baby Record 2014 Evidence-based tool for the health of infants and children from birth to age 5. Can Fam Physician 2015;61(11):949-955.

3. Greenhawt MJ, Fleischer DM, Atkins D, Chan ES. The Complexities of Early Peanut Introduction for the Practicing Allergist. J Allergy Clin Immunol Pract 2016;4(2):221-225.

4. Fleischer DM, Spergel JM, Assa'ad AH, Pongracic JA. Primary prevention of allergic disease through nutritional interventions. J Allergy Clin Immunol Pract 2013;1(1):29-36.

5. Alm B, Åberg N, Erdes L, Möllborg P, Pettersson R, Norvenius $\mathrm{SG}$ et al. Early introduction of fish decreases the risk of eczema in infants. Arch Dis Child 2009;94(1):11-15.

6. Sicherer SH, Muñoz-Furlong A, Sampson HA. Prevalence of peanut and tree nut allergy in the United States determined by means of a random digit dial telephone survey: a 5-year follow-up study. J Allergy Clin Immunol 2003;112(6):12031207.

7. Thomsen SF. Epidemiology and natural history of atopic diseases. Eur Clin Respir J 2015;2(1):24642.

8. Chan ES, Cummings C, Atkinson A, Chad Z, Francoeur MJ, Kirste L et al. Dietary exposures and allergy prevention in high-risk infants: a joint position statement of the Canadian Society of Allergy and Clinical Immunology and the Canadian Paediatric Society. Allergy Asthma Clin Immunol 2014;10(1):1.

9. Sampson HA, Aceves S, Bock SA, James J, Jones S, Lang D et al. Food allergy: a practice parameter update-2014. J Allergy Clin Immunol 2014;134(5):1016-1025.

10. Du Toit G, Roberts G, Sayre PH, Bahnson HT, Radulovic S, Santos AF et al. Randomized trial of peanut consumption in infants at risk for peanut allergy. N Engl J Med 2015;372(9):803813.

11. Fleischer DM, Sicherer S, Greenhawt M, Campbell D, Chan ES, Muraro A et al. Consensus communication on early peanut introduction and the prevention of peanut allergy in high-risk infants. World Allergy Organ J 2015;8(1):1.

12. Koplin JJ, Allen KJ, Gurrin LC, Peters RL, Lowe AJ, Tang ML et al. The impact of family history of allergy on risk of food allergy: a population-based study of infants. Int J Environ Res Public Health 2013;10(11):5364-5377.

13. Nwaru BI, Takkinen HM, Niemelä O, Kaila M, Erkkola M, Ahonen $\mathrm{S}$ et al. Introduction of complementary foods in infancy and atopic sensitization at the age of 5 years: timing and food diversity in a Finnish birth cohort. Allergy 2013;68(4):507-516.

14. Du Toit G, Katz Y, Sasieni P, Mesher D, Maleki SJ, Fisher HR et al. Early consumption of peanuts in infancy is associated with a low prevalence of peanut allergy. J Allergy Clin Immunol 2008;122(5):984-991.

15. Poole JA, Barriga K, Leung DY, Hoffman M, Eisenbarth GS, Rewers $M$ et al. Timing of initial exposure to cereal grains and the risk of wheat allergy. Pediatrics 2006;117(6):2175-2182.

16. Fox AT, Sasieni P, du Toit G, Syed H, Lack G. Household peanut consumption as a risk factor for the development of peanut allergy. J Allergy Clin Immunol 2009;123(2):417-423.

17. Abrams EM, Becker AB. Food introduction and allergy prevention in infants. CMAJ 2015;187(17):1297.

18. Burks AW, Laubach S, Jones SM. Oral tolerance, food allergy, and immunotherapy: implications for future treatment. J Allergy Clin Immunol 2008;121(6):1344-1350. 